\title{
Теоретическое моделирование термоэлектрических свойств сплавов Гейслера $\mathrm{Fe}_{2} \mathrm{Ti}_{1-x} \mathrm{~V}_{x} \mathrm{Sn}$
}

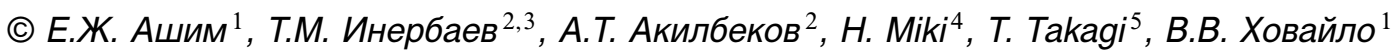 \\ ${ }^{1}$ Национальный исследовательский технологический университет „МИСиС“, \\ 119049 Москва, Россия \\ ${ }^{2}$ Евразийский национальный университет им. Л.Н. Гумилева, \\ 010000 Астана, Казахстан \\ ${ }^{3}$ Институт геологии и минералогии им. В.С. Соболева \\ Сибирского отделения Российской академии наук, \\ 630090 Новосибирск, Россия \\ ${ }^{4}$ Frontier Research Institute for Interdisciplinary Science Tohoku University, \\ Sendai 980-8578, Japan \\ ${ }^{5}$ Institute of Fluid Sciences, Tohoku University, \\ Sendai 980-8577, Japan \\ E-mail: ye.ashim.misis@gmail.com
}

Поступила в Редакцию 6 марта 2019 г.

В окончательной редакции 11 марта 2019 г.

Принята к публикации 11 марта 2019 г.

Приведены теоретические расчеты электронной структуры и коэффициента Зеебека в сплавах $\mathrm{Fe}_{2} \mathrm{Ti}_{1-x} \mathrm{~V}_{x} \mathrm{Sn}$ для случаев полностью упорядоченной $L 2_{1}$ и частично разупорядоченной $B 2$ кристаллической структуры Гейслера. Показано, что ширина запрещенной зоны увеличивается при замещении Ti на V. Сравнение с доступными теоретическими и экспериментальными данными указывает на то, что учет хаотичности в распределении атомов позволяет получить значения коэффициента Зеебека, более близкие к результатам экспериментов.

DOI: $10.21883 /$ FTP.2019.07.47860.40

\section{1. Введение}

Теоретическая оценка свойств термоэлектрических материалов является необходимым звеном при поиске перспективных термоэлектрических материалов (ТЭМ). Оценка перспективности ТЭМ может быть дана с помощью термоэлектрической добротности - безразмерной величины, которая определяется по формуле [1]

$$
Z T=S^{2} \sigma T / \kappa
$$

где $S$ - коэффициент Зеебека (термоэдс), $\sigma$ - электропроводность, $T-$ температура, $\kappa$ - теплопроводность. В данной работе основное внимание уделяется расчетам коэффициента Зеебека, который дает больший вклад в термоэлектрическую добротность материала.

$\mathrm{Fe}_{2} \mathrm{TiSn}$ - это сплав Гейслера с 24 валентными электронами [2]. Стандартный анализ положения пиков в рентгеновской дифракционной картине показал, что он кристаллизуется в кубическую структуру типа $L 22_{1}$, с параметром решетки $6.074 \AA$ [3]. Кристаллическую структуру сплавов Гейслера можно представить как образованную четырьмя взаимопроникающими ГЦК-подрешетками, расположение которых определяется позициями (в координатах Вайкоффа) $A(1 / 41 / 41 / 4), B(3 / 43 / 43 / 4), C \quad\left(\begin{array}{lll}0 & 0 & 0\end{array}\right)$ и $D(1 / 21 / 21 / 2)$ [4]. Элементарная ячейка содержит 16 атомов, по 4 на каждую подрешетку, из которых позиции $A, B$ занимают атомы железа, позиции $C$ и
$D$ занимают атомы олова и титана соответственно. Ранее группа исследователей во главе с Шином Ябуучи (Shin Yabuuchi) [5] предсказала оптимальный уровень замещения $x=0.06$ в сплаве Гейслера $\mathrm{Fe}_{2} \mathrm{Ti}_{1-x} \mathrm{~V}_{x} \mathrm{Sn}$ для увеличения термоэдс и коэффициента мощности. Предыдущие расчеты [5] выполнены для элементарной ячейки стехиометрического $\mathrm{Fe}_{2} \mathrm{TiSn}$ без замещения атомов титана на атомы ванадия. Чтобы восполнить этот пробел, в данной работе первопринципные расчеты были проведены для структур с уровнями замещения $x=0.0625,0.125,0.25$ в $\mathrm{Fe}_{2} \mathrm{Ti}_{1-x} \mathrm{~V}_{x} \mathrm{Sn}$.

\section{2. Методика вычислений}

Первопринципные вычисления в рамках теории функционала плотности выполнены с помощью программы Vienna $a b$ initio Simulation Package (VASP) [6,7], с использованием метода проецированных присоединенных плоских волн (projector augmented wave method) [8], энергия обрезания - 500 эВ. Использовано приближение обменного корреляционного функционала ПердьюБурке-Эрнзерхоффа [9]. Релаксация атомов в ячейках, а также оптимизация параметра решетки с равномерным, идеальным распределением атомов (структура типа $L 2_{1}$ ) проводилась с использованием $15 \times 15 \times 15 k$-точек. Кристаллическая структура с хаотическим распределением атомов в сплаве, которая соответствует частично разупорядоченной структуре типа $B 2$ (рис. 1), опти- 
Детали вычислений зонной структуры $\mathrm{Fe}_{2} \mathrm{Ti}_{1-x} \mathrm{~V}_{x} \mathrm{Sn}(x=0$, $0.0625,0.125,0.25)$ для случая $L 2_{1}$ структуры

\begin{tabular}{l|c|c|c}
\hline $\begin{array}{c}\text { Уровень } \\
\text { замещения, } x\end{array}$ & $\begin{array}{c}\text { Суперьячейка, } \\
\text { (число атомов) }\end{array}$ & $\begin{array}{c}\text { Замещенных } \\
\text { атомов Тi } \rightarrow \mathrm{V}\end{array}$ & $\begin{array}{c}\text { Решетка } \\
\text { из } k \text {-точек }\end{array}$ \\
\hline 0 & Элементарная (16) & 0 & $60 \times 60 \times 60$ \\
0.0625 & $2 \times 2 \times 1(64)$ & 1 & $20 \times 20 \times 40$ \\
0.125 & $2 \times 1 \times 1(32)$ & 1 & $20 \times 40 \times 40$ \\
0.25 & Элементарная (16) & 1 & $40 \times 40 \times 40$
\end{tabular}

мизировалась с использованием $2 \times 2 \times 2 k$-точек. При оптимизации суперьячеек $2 \times 1 \times 1$ и $2 \times 2 \times 1$ был использован параметр KSPACING $=0.1$, определяющий сетку в $k$-пространстве.

Уровни замещения $x=0.0625,0.125,0.25$ в $\mathrm{Fe}_{2} \mathrm{Ti}_{1-x} \mathrm{~V}_{x} \mathrm{Sn}$ были достигнуты трансляцией элементарной ячейки и замещением определенного числа атомов Ti на $\mathrm{V}$, как указано в таблице, во втором и третьем столбцах. В последнем столбце указана размерность сетки в $k$-пространстве. Для оценки влияния разупорядоченного расположения атомов, которое соответствует структуре типа $B 2$, а также наличия вакансий и дефектов упаковки на коэффициент Зеебека были рассмотрены суперъячейки $2 \times 2 \times 2$ (128 атомов) с близкими к предыдущим уровнями замещения $(x=0.065,0.129,0.26)$, с добавлением вакансии на позицию олова (3\%), а также с добавлением дефектов упаковки (3\%); атомы ванадия в суперьячейках распределены хаотично (см. рис. 1). Для определения зонной структуры была выбрана сетка $10 \times 10 \times 10$ $k$-точек. Вычисление коэффициента Зеебека выполнено с использованием пакета программ BoltzTraP [10], в приближении постоянного времени релаксации.

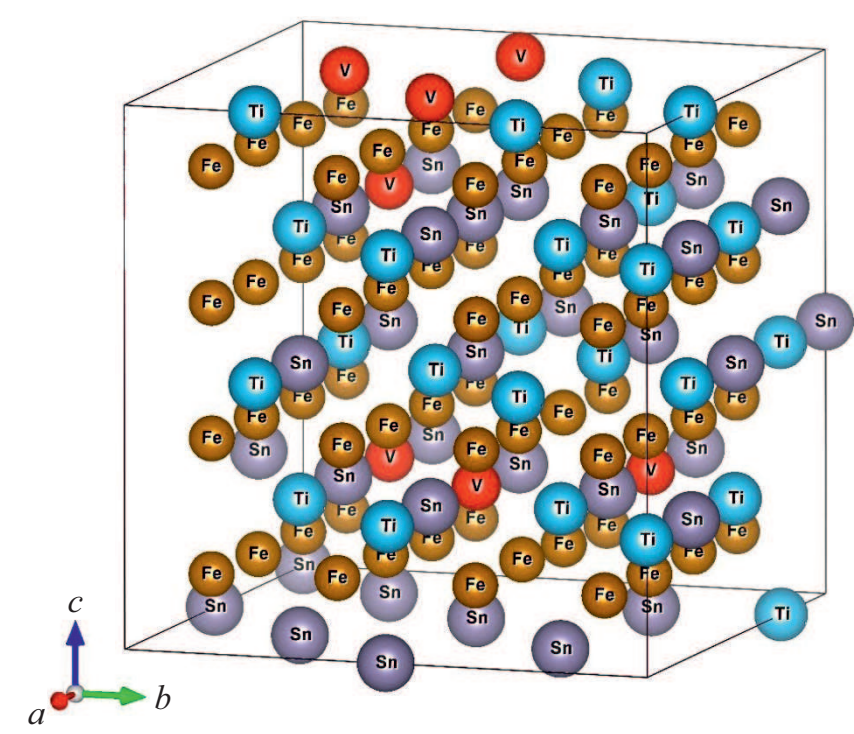

Рис. 1. Суперъячейка $2 \times 2 \times 2 \mathrm{Fe}_{2} \mathrm{Ti}_{1-x} \mathrm{~V}_{x} \mathrm{Sn}(x=0.25)$ типа $B 2$ с неравномерным распределением атомов ванадия.

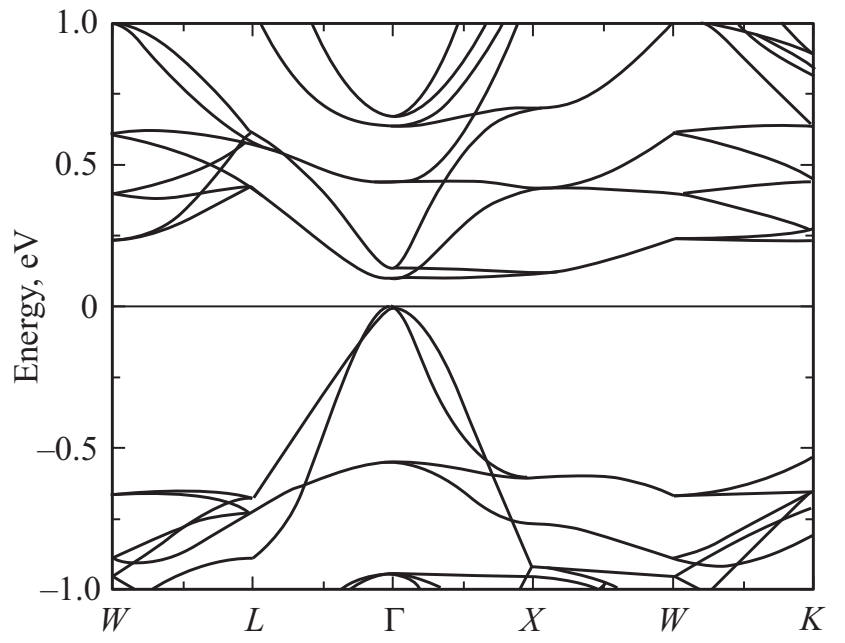

Рис. 2. Зонная структура $\mathrm{Fe}_{2} \mathrm{TiSn}$ вдоль точек высокой симметрии $W-L-\Gamma-X-W-K$ в зоне Бриллюэна.

\section{3. Результаты вычислений}

Для стехиометрического сплава Гейслера $\mathrm{Fe}_{2} \mathrm{TiSn}$ параметр кристаллической решетки после оптимизации был найден равным $a=6.0316 \AA$, что сопоставимо с результатами предыдущих теоретических $(a=6.032 \AA[5])$ и экспериментальных $(a=6.074 \AA$ [3] $)$ исследований. Для уровней легирования $x=0.0625, x=0.125$ и $x=0.25$ параметры кристаллической решетки равны соответственно 6.027, 6.022 и $6.014 \AA$. Вычисление электронной зонной структуры показало (рис. 2), что во всех образцах мы имеем дело с прямозонным полупроводником. Ширина запрещенной зоны составила 0.107 эВ для нелегированного сплава, а для уровней легирования $x=0.0625, x=0.125, x=0.25$ ширина запрещенной зоны равна $0.111,0.114$ и 0.133 эВ соответственно. Видно, что изменение ширины запрещенной зоны коррелирует с изменением постоянной решетки. На рис. 3 приведены парциальные плотности состояний нелегированного $\mathrm{Fe}_{2} \mathrm{TiSn}$, а на рис. 4 - для $\mathrm{Fe}_{2} \mathrm{Ti}_{1-x} \mathrm{~V}_{x} \mathrm{Sn}$ $(x=0.25)$. Локальная парциальная плотность состояний на каждый атом $\mathrm{Fe}_{2} \mathrm{TiSn}$ (рис. 3) сопоставима с предыдущими вычислениями, в частности с источником [11]. В случае с $\mathrm{Fe}_{2} \mathrm{Ti}_{1-x} \mathrm{~V}_{x} \mathrm{Sn}(x=0.25$, рис. 4) наблюдается влияние привнесенных ванадием состояний на полную плотность состояний. Из визуализации высших занятых (highest occupied molecular orbital, HOMO) и низших незанятых (lowest unoccupied molecular orbital, LUMO) молекулярных орбиталей видно, что при переходе $\mathrm{HOMO} \rightarrow$ LUMO в случае нелегированного $\mathrm{Fe}_{2} \mathrm{TiSn}$ (см. вставки на рис. 3) не наблюдается значительной электронной плотности в плоскости (100), отстоящей от начала координат на $0.5 a$, тогда как в случае $\mathrm{Fe}_{2} \mathrm{Ti}_{1-x} \mathrm{~V}_{x} \mathrm{Sn}(x=0.25$, см. вставки в рис. 4) видно, что в этой плоскости появляется определенное распределение электронной плотности, локализованной около атома ванадия. 


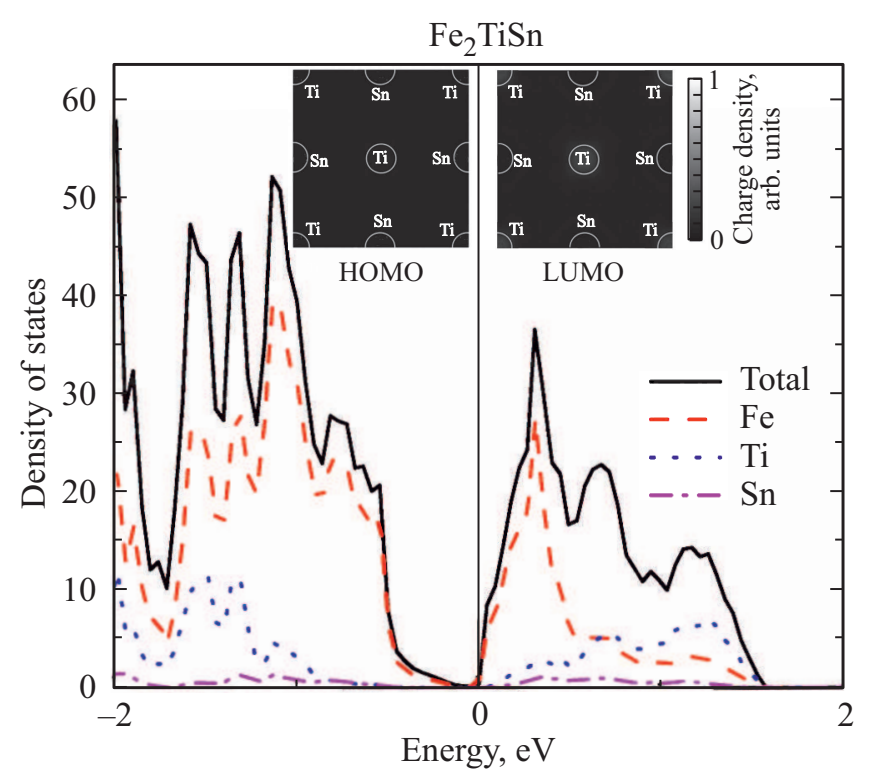

Рис. 3. Парциальная плотность состояний $\mathrm{Fe}_{2} \mathrm{TiSn}$, сплошной вертикальной линией обозначен уровень Ферми, слева и справа от нее показаны HOMO и LUMO соответственно.

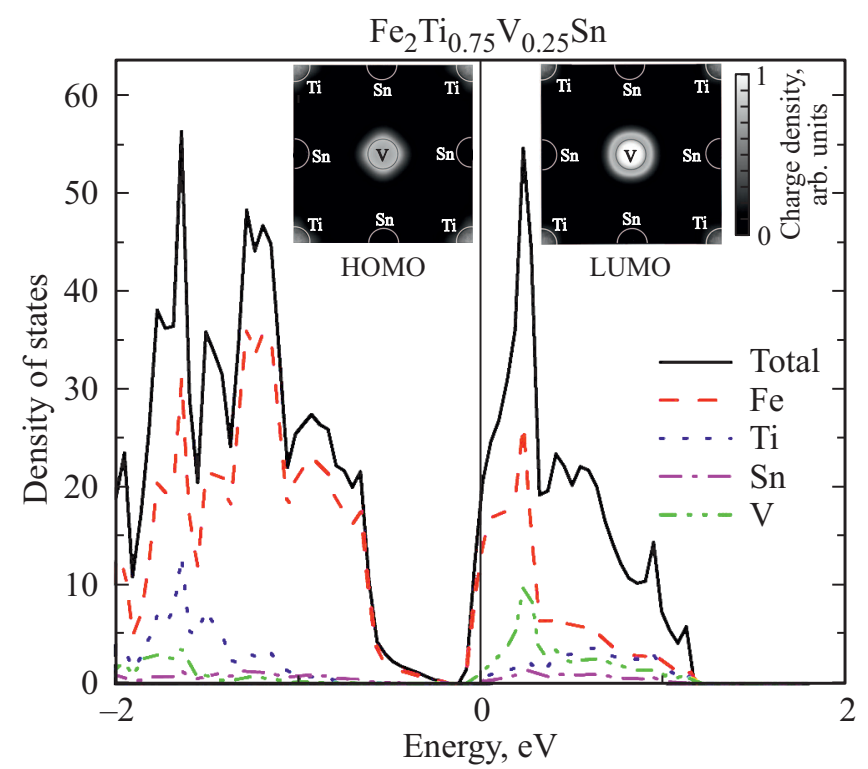

Рис. 4. Парциальная плотность состояний $\mathrm{Fe}_{2} \mathrm{Ti}_{1-x} \mathrm{~V}_{x} \mathrm{Sn}$, сплошной вертикальной линией обозначен уровень Ферми, слева и справа от нее показаны HOMO и LUMO соответственно.

В случае полностью упорядоченной $L 2_{1}$ структуры и равномерного распределения атомов ванадия наблюдается смена знака коэффициента Зеебека с „ $+^{“}$ при нелегированном $\mathrm{Fe}_{2} \mathrm{TiSn}$ на „,-“ при всех уровнях легирования $\mathrm{Fe}_{2} \mathrm{Ti}_{1-x} \mathrm{~V}_{x} \mathrm{Sn}$, который демонстрирует по абсолютным значениям десятикратный рост $S$ по сравнению с нелегированным $\mathrm{Fe}_{2} \mathrm{TiSn}$, что согласуется с выводом предыдущего теоретического исследования этого сплава [5]. Однако при сравнении с результатами эксперименталь- ного исследования аналогичных сплавов Гейслера [12] со схожими уровнями замещения $\mathrm{Ti}$ на $\mathrm{V}$, показанными на рис. 5, очевидно, что применяемый метод вычислений сильно переоценивает влияние замещения титана на ванадий на термоэлектрические свойства $\mathrm{Fe}_{2} \mathrm{Ti}_{1-x} \mathrm{~V}_{x} \mathrm{Sn}$.

Результаты вычислений для случая частично разупорядоченной структуры типа $B 2$ и неравномерного распределения атомов $\mathrm{V}$ в суперьячейке (рис. 1) более реалистичны. Из рис. 6 можно заключить, что коэффици-

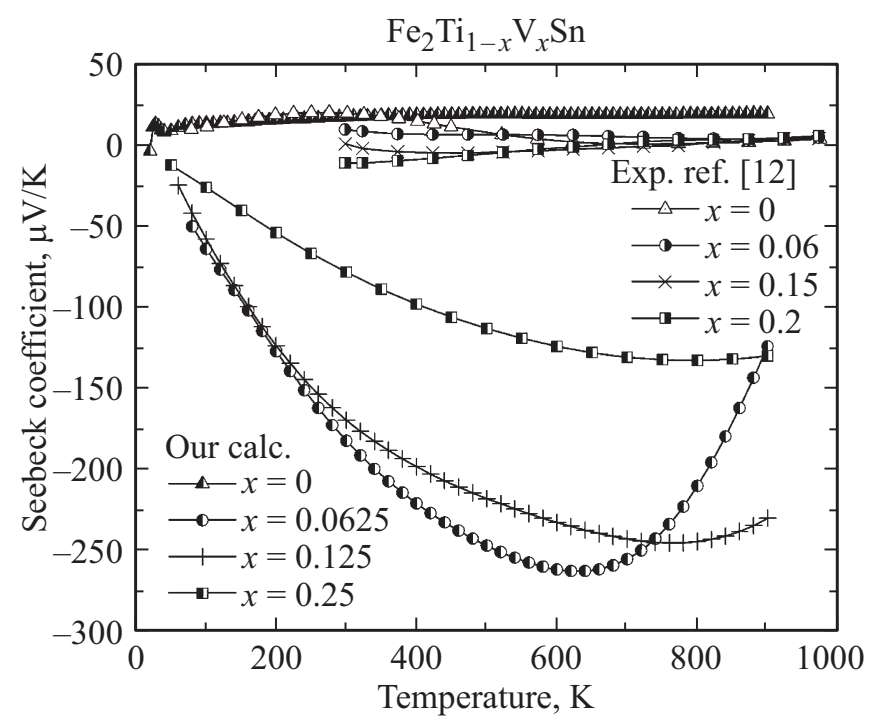

Рис. 5. Зависимость коэффициентов Зеебека от температуры для сплавов Гейслера $\mathrm{Fe}_{2} \mathrm{Ti}_{1-x} \mathrm{~V}_{x} \mathrm{Sn}(x=0,0.0625,0.125,0.25)$ с идеальным распределением атомов. Сравнительные экспериментальные кривые с уровнями замещения титана $x=0,0.06$, $0.15,0.2$ взяты из источника [12].

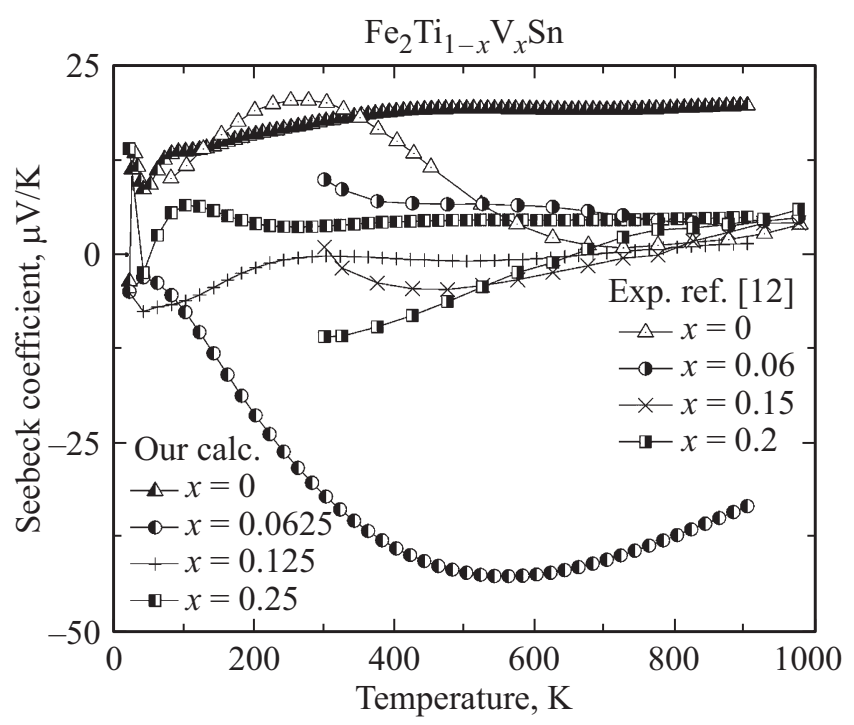

Рис. 6. Зависимость коэффициентов Зеебека от температуры для сплавов Гейслера $\mathrm{Fe}_{2} \mathrm{Ti}_{1-x} \mathrm{~V}_{x} \mathrm{Sn}(x=0,0.0625,0.125,0.25)$ с хаотическим распределением атомов. Сравнительные экспериментальные кривые с уровнями замещения титана $x=0$, $0.06,0.15,0.2$ взяты из работы [12]. 
ент Зеебека в лучшем случае достигает -42 мкВ/K, что в 5 раз меньше по модулю чем $S$, вычисленный для $L 2_{1}$ структуры с равномерным распределением ванадия. При неравномерном распределении атомов в суперьячейке наилучшим уровнем замещения для повышения термоэдс является $x=0.0625$, что соответствует предсказаниям предыдущего теоретического исследования [5].

\section{4. Заключение}

В данной работе оценены коэффициенты Зеебека для сплавов Гейслера $\mathrm{Fe}_{2} \mathrm{Ti}_{1-x} \mathrm{~V}_{x} \mathrm{Sn}(x=0,0.0625,0.125$, $0.25)$. Вычисления показывают для $x=0$ результаты, относительно близкие к экспериментальным. При применении того же метода вычислений к образцам с равномерным распределением ванадия, несмотря на сходство и подтверждение предыдущих теоретических вычислений, результаты сильно отличаются от экспериментальных.

Существенное отличие теоретических вычислений и результатов эксперимента можно объяснить тем, что теория не учитывает неидеальность экспериментальных образцов. Расчет с учетом дефектов упаковки, вакансий и неравномерного распределения атомов ванадия в суперьячейке позволяет получить значения термоэдс, более близкие к экспериментальным данным.

\section{Финансирование работы}

Работа поддержана Российским фондом фундаментальных исследований (грант № 18-52-45018). Часть исследований выполнена в рамках совместного исследовательского проекта с Институтом науки о жидкостях Университета Тохоку. Вычисления были частично выполнены на суперкомпьютерном кластере „Cherry“, предоставленном Лабораторией моделирования и разработки материалов в НИТУ „МИСиС“ (поддерживается грантом Министерства образования и науки Российской Федерации № 14.Y26.31.0005). Часть работы поддержана Министерством образования и науки Республики Казахстан, договор № 132 от 12 марта 2018 г. „Дизайн перспективных термоэлектрических полупроводниковых материалов методами расчета из первых принципов“" по приоритету „Энергетика и машиностроение“.

\section{Список литературы}

[1] G.A. Slack. CRC Handbook of Thermoelectrics (CRC Press, 1995).

[2] I. Galanakis, P.H. Dederichs, N. Papanikolaou. Phys. Rev. B, 66 (17), 174429 (2002).

[3] P.I. Kripyakevich, V.Ya. Markiv. Dopovidi Akademii Nauk Ukrains'koi RSR, 1606 (1963).

[4] A. Slebarski. J. Phys. D: Appl. Phys., 39 (5), 856 (2006).

[5] S. Yabuuchi, M. Okamoto, A. Nishide, Y. Kurosaki, J. Hayakawa. Appl. Phys. Express, 6 (2), 025504 (2013).

[6] G. Kresse, J. Furthmuller. Phys. Rev. B, 54 (16), 11169 (1996).
[7] G. Kresse, D. Joubert. Phys. Rev. B, 59 (3), 1758 (1999).

[8] P.E. Blochl. Phys. Rev. B, 50 (24), 17953 (1994).

[9] J.P. Perdew, K. Burke, M. Ernzerhof. Phys. Rev. Lett., 77 (18), 3865 (1996).

[10] G.K.H. Madsen, D.J. Singh. Comput. Phys. Commun., 175 (1), 67 (2006).

[11] X. Bin, Y. Lin. J. Phys. D: Appl. Phys., 41 (9), 095404 (2008).

[12] А.А. Таранова, А.П. Новицкий, А.И. Воронин, С.В. Таскаев, Т. Takagi, Н. Miki, В.В. Ховайло. ФТП, 53 (6), 777 (2019).

Редактор Г.А. Оганесян

\section{Theoretical modeling of thermoelectric properties of $\mathrm{Fe}_{2} \mathrm{Ti}_{1-x} \mathbf{V}_{x} \mathrm{Sn}$ Heusler alloys}

Ye.Zh. Ashim 1, T.M. Inerbaev, 2,3, A.T. Akilbekov², H. Miki ${ }^{4}$, T. Takagi ${ }^{5}$, V.V. Khovaylo ${ }^{1}$

${ }^{1}$ National Research Technological University „MISiS“, 119049 Moscow, Russia

2 Gumilyov Eurasian National University, 010000 Astana, Kazakhstan

${ }^{3}$ Sobolev Institute of Geology and Mineralogy, Siberian Branch of the Russian Academy of Sciences, 630090 Novosibirsk, Russia

${ }^{4}$ Frontier Research Institute

for Interdisciplinary Science, Tohoku University,

Sendai 980-8578, Japan

${ }^{5}$ Institute of Fluid Sciences, Tohoku University, Sendai 980-8577, Japan

Abstract This paper presents theoretical calculations of electronic structure and Seebeck coefficient in $\mathrm{Fe}_{2} \mathrm{Ti}_{1-x} \mathrm{~V}_{x} \mathrm{Sn}$ alloys for the cases fully ordered $L 2_{1}$ and partially disordered $B 2$ Heusler crystal structure. It is shown that the band gap increases with the $\mathrm{Ti}$ by $\mathrm{V}$ substitution. Comparison with the available theoretical and experimental data indicates that taking into account the randomness in the distribution of atoms makes it possible to obtain more comparable to the experimental data values of the Seebeck coefficient 